\title{
Uma proposta de ensino de desenho em design gráfico a partir de uma abordagem projetual de sistemas de wayfinding
}

\author{
A proposal for teaching drawing in graphic design based on a design process of \\ wayfinding systems
}

\section{Anelise Zimmermann \& Solange Coutinho}

desenho, design gráfico, design da informação, wayfinding, ensino

\begin{abstract}
Esse artigo apresenta parte dos resultados da pesquisa "O ensino e a prática do desenho na formação em Design Gráfico", dando ênfase ao estudo de métodos projetuais de sistemas de wayfinding por meio de práticas em desenho. Este estudo dá sequência à tese de Zimmermann (2016) que propõe um modelo de ensino do desenho em Design Gráfico a partir de uma abordagem interdisciplinar e projetual. A pesquisa caracteriza-se como qualitativa, exploratória e descritiva. Para a coleta dos dados foram utilizados a pesquisa bibliográfica e o estudo de campo, tendo como instrumentos o diário de campo e a digitalização de desenhos produzidos pelos alunos participantes. Os dados coletados concentram-se especificamente nas atividades desenvolvidas em aula referentes ao desenho de mapas para sistemas de wayfinding de baixa complexidade. Para o desenvolvimento dessas atividades, tomou-se como base 0 método projetual desenvolvido pelo estúdio Applied Wayfinding e aplicado aos projetos Legible London e Walk Rio. Como resultado, identificou-se o campo do design de informação como uma das possíveis áreas geradoras de conteúdos para o ensino de disciplinas de desenho, permitindo explorar formas e funções do desenhar a partir de métodos projetuais, aproximando disciplinas do currículo entre si e a práticas profissionais.
\end{abstract}

drawing, graphic design, information design, wayfinding, teaching

This paper presents some of the results of the research entitled "The teaching and practice of drawing on a graphic design course", emphasizing the study of wayfinding systems through drawing practices. This study follows up the thesis of Zimmermann (2016) which proposes a model for teaching drawing on graphic design courses based on an interdisciplinary approach linked to foundations of the design process. The research is classified as being qualitative, exploratory and descriptive, and includes a bibliographic and field study. The instruments used to collect the data were a field diary and digitalized copies of the drawings produced by the students. The collected data specifically concentrates on the activities developed during classes involving drawing maps for low complexity wayfinding systems. The activities were based on the design method developed by the Applied Wayfinding studio, which guided the development of Legible London's and Walk Rio's wayfinding projects. As a result, this paper identifies that the information design field may generate a diversity of topics and contents for teaching drawing, thereby enabling the exploration of its diverse roles throughout the design process. Moreover, this study also helped to establish links among subjects of design teaching and professional practices.

\section{Introdução}

Esse artigo apresenta parte dos resultados da pesquisa "O ensino e a prática do desenho na formação em Design Gráfico" realizada no período de 2017 e 2018 no Curso de Design Gráfico da Universidade do Estado de Santa Catarina, dando ênfase ao estudo de métodos projetuais de sistemas de wayfinding por meio do ensino e práticas do desenho. Essa pesquisa dá sequência à tese de Zimmermann (2016), a qual propõe um modelo de ensino do desenho em Design Gráfico a partir de uma abordagem interdisciplinar e projetual. Tal abordagem sugere um diálogo com as demais disciplinas do currículo e com o meio profissional, observando as diversas formas, aplicações e funções desenhar. Aqui entende-se o desenho de forma ampla, compreendendo sua aplicação não apenas como um fim em si mesmo, mas também como um

Anais do 9 $\mathrm{CIDI}$ e 9 CONGIC

Luciane Maria Fadel, Carla Spinillo, Anderson Horta, Cristina Portugal (orgs.)

Sociedade Brasileira de Design da Informação SBDI

Belo Horizonte | Brasil | 2019

ISBN 978-85-212-1728-2
Proceedings of the 9th CIDI and 9th CONGIC

Luciane Maria Fadel, Carla Spinillo, Anderson Horta,

Cristina Portugal (orgs.)

Sociedade Brasileira de Design da Informação SBDI

Belo Horizonte | Brazil | 2019

ISBN 978-85-212-1728-2 
meio, o que inclui tanto o desenho finalizado de mapas, diagramas e infográficos, como os esboços produzidos para a compreensão de espaços, registro de ideias e organização de informações de forma individual e coletivamente. Já a abordagem interdisciplinar e projetual diz respeito ao reestabelecimento de aproximação entre áreas e conhecimentos, que em sua essência, estão interligados. Considerando tais aspectos, o objetivo deste estudo foi implementar o modelo de ensino de desenho apresentado na tese, tendo como foco a aproximação entre o ensino e prática do desenho e o design de informação.

Outras experiências de ensino do desenho a partir do processo projetual já foram relatadas anteriormente por Zimmermann (2016) e Zimmermann e Coutinho (2018), como as atividades realizadas por Schaeverbeke e Heylighen (2012) na Faculdade de Arquitetura (Bélgica) e o método proposto por Cabau (2011), aplicado nos cursos de Arquitetura de Design de Produto da Escola Superior de Artes e Design das Caldas da Rainha (Portugal). Entretanto, de uma forma geral, as publicações para o ensino do desenho de caráter didático referem-se, em sua grande maioria, ao ensino de aspectos técnicos voltados principalmente ao desenho geométrico ou artístico.

Assim sendo, a relevância dessa pesquisa se dá quanto ao registro da implementação do modelo proposto em situações reais de ensino e direcionada ao estudo do desenho por meio do design de informação a partir de sistemas de wayfinding. Também se inclui nesse estudo, a análise, avaliação a verificação da necessidade de ajustes do modelo de ensino de desenho proposto.

\section{0 desenho no design da informação}

Para essa pesquisa adotou-se a definição de desenho elaborada por Farthing (2011) a qual diz que o desenho, assim como as palavras, os números e as notações (musicais, por exemplo), é a tradução de eventos multidimensionais em informações bidimensionais compreensíveis. Por eventos multidimensionais entendem-se eventos que podem existir fisicamente ou não. Já, o termo compreensível considera o contexto no qual o desenho foi feito e ao qual observador se dirige.

Essa definição abrange o desenho de forma ampla e relaciona-se à necessidade humana da comunicação, do registro, de construir e reconstruir o mundo físico ou o que se passa na imaginação, sem fazer menção à determina área do conhecimento, técnica, material ou suporte. Assim, inclui-se aqui, com a mesma importância, os desenhos de esboços, desenhos técnicos, diagramas, ilustrações, mapas, desenhos geométricos, entre tantos outros. É o uso e o contexto de cada um desses tipos de desenho que lhe dá seu valor.

A essa definição soma-se a classificação da linguagem visual de Twyman (1985) a qual divide-se em não-gráfica e gráfica, com as subdivisões verbal, pictórica e esquemática (fig. 1).

Figura 1: Classificação da linguagem visual e linguagem visual gráfica por Twyman (1982). (Fonte: adaptado pelas autoras).

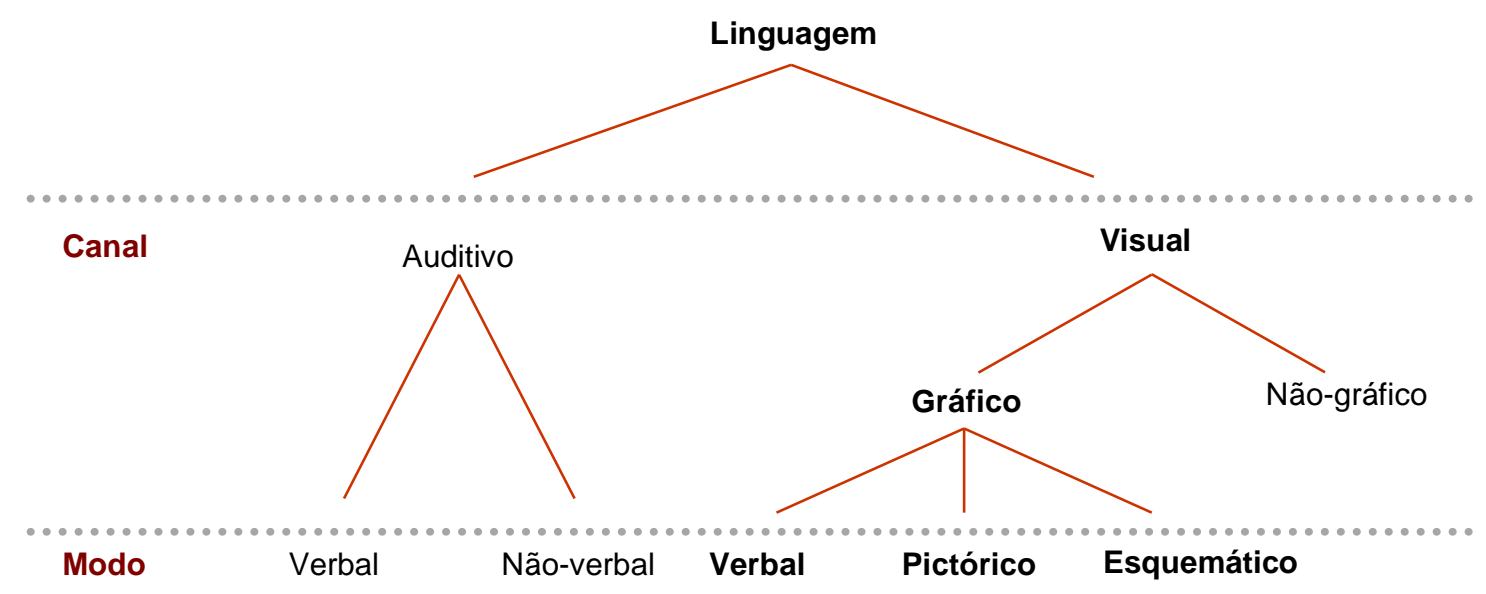

Anais do 9o Congresso Internacional de Design da Informação | CIDI 2019 
A linguagem visual verbal refere-se, por exemplo, à caligrafia, o letreiramento, à escrita ou à tipografia. Já a pictórica, pode ser exemplificada por ilustrações, fotografias e pictogramas. De acordo com Twyman (1982), a linguagem pictórica refere-se a alguma imagem que se relaciona mesmo que distante com a aparência ou estrutura de alguma coisa real ou imaginada.

Por fim, a linguagem esquemática refere-se a elementos que integram mensagens visuais e que não se enquadram às demais categorias (verbal ou pictórica), como as setas e formas empregadas em diagramas. Os três modos do uso da linguagem, segundo o autor (op. cit.), são independentes da forma de execução, se produzidos à mão ou por meio de algum instrumento, mecanismo ou máquina. Com base nesses aspectos, considera-se que diagramas e setas são também desenhos, pois representam elementos tridimensionais por meio de linhas. O desenho da seta guarda relação com a flecha e indica não apenas o objeto, como também a ação que executa, movimento e direção, inserido em um espaço tridimensional' ${ }^{1}$.

Essa compreensão de desenho, ao se aproximar da palavra, dos números e das notações, expõe o caráter interdisciplinar do desenho, que tanto transita por diferentes áreas, como as aproxima. É como base nesses aspectos, entre outros, que o modelo de ensino de desenho proposto por Zimmermann (2016) se fundamenta na possibilidade de explorar o ensino e a prática do desenho como uma forma de aproximar conteúdos, disciplinas e metodologias em design gráfico.

Tais ideias partem da concepção de processo e do exercício do desenhar a partir de objetivos variados. No campo do Design da Informação, essas funções incluem não apenas as representações que compõem os projetos finais, como os pictogramas finalizados, mas também incorporam os desenhos que contribuem no processo de organização da informação, como os diagramas, gráficos e mapas, os desenhos de investigação ou de geração de ideias, como os esboços.

Convém destacar que Cross (2011) chama a atenção para a importância do desenho de esboços no processo projetual pois este permite que muitos aspectos de um mesmo projeto sejam facilmente trabalhados de forma simultânea, envolvendo diferentes níveis de abstração. Essa flexibilidade do esboço também oferece a possibilidade de segmentação do problema e agrupamento de suas partes em diferentes formas. Assim, durante o processo projetual o esboço permite que sejam realizados diversos testes de muitas combinações, antecipando problemas de forma rápida e sem grande investimento técnico. Goldschmidt (1991) e Goel (1999) também discorrem sobre o desenho de esboços a partir da característica da ambiguidade, muitas vezes presente nos desenhos feitos de forma rápida, sem grande preocupação pictórica. Essa característica é apontada como significativa no estímulo de processos de geração alternativas, podendo ampliar as formas de se observar um problema. Para esses pesquisadores, esse tipo de desenho corresponde a uma forma particular de raciocínio: o pensar desenhando.

Assim, conhecer as propriedades e recursos dos diversos tipos de desenho permite aos designers explorá-los de forma eficiente e adequada aos objetivos e etapas de projetos, tendose em consideração que a elaboração de desenhos com objetivos informacionais envolve a mesma complexidade que a composição de mensagens verbais. Assim, parte-se da estruturação de dados brutos que precisam ser transformados em informações visuais relevantes e significativas. Para David Sless (1982) o design da informação é a área que se encarrega do gerenciamento da relação entre pessoas e a informação de forma que a torne acessível e usável. Da mesma forma como a escolha de cada palavra ou uma vírgula pode interferir na compreensão do texto verbal em uma mensagem, o mesmo se dá quanto aos elementos visuais que compõem um desenho. As escolhas são sempre significativas e participam de ações e tomada de decisões de seus usuários, como na direção adotada a partir de elementos em um desenho de mapa ou de um pictograma em uma parede.

\footnotetext{
${ }^{1}$ Na perspectiva de Twyman (1979) a fronteira entre os modos de simbolização da linguagem pictórica e esquemática é tênue. Por exemplo, se o leitor compreender a seta como o objeto 'flecha', então esse elemento significará para ele como o modo pictórico, todavia se o leitor compreender esse elemento como um indicativo de movimento ou direção, será para ele como o modo esquemático.
} 


\section{0 ensino do desenho em Design Gráfico - uma proposta a partir de sistemas de wayfinding}

A atividade de desenho analisada nessa pesquisa consistiu no estudo e prática do desenho a partir de exemplos de projetos de wayfinding, especificamente os trabalhos Legible London e Walk Rio. Esses projetos foram desenvolvidos pelo estúdio Applied Wayfiding sob a coordenação de Tim Fendley. O processo de concepção dos projetos foi relatado em entrevista registrada em filme (O desenho conectando conhecimentos. Episódio 6: O desenho no Design de Informação, 2018²). Nessa entrevista, Fendley aborda o método de projeto utilizado, com ênfase na importância e formas de uso do desenho em suas diferentes etapas. Nesses casos, o uso do desenho assumiu diversas funções, sendo elas: estudo dos espaços físicos por meio de esboços; estudos de sequência de possíveis ações dos usuários; organização das informações; apresentação das informações aos clientes; troca de informações por meio de desenhos coletivos; desenhos dos mapas e estudos de formas de representação dos espaços tridimensionais; estudos dos suportes; estudos de interação entre sinalização, ambiente e usuário e desenhos técnicos para a produção. A descrição técnica do projeto Legible London é também apresentada na publicação Yellow Book (Applied Information Group, 2007).

Como base nesse conteúdo foi elaborada uma atividade de desenho inserida na disciplina de Laboratório de Desenho II, que integra o projeto pedagógico do Curso de Design Gráfico do Universidade do Estado de Santa Catarina (UDESC) na segunda fase do curso, sendo aplicada nos anos de 2017 e 2018. A disciplina de Laboratório de Desenho I/ tem como objetivo o estudo e prática do desenho direcionado especificamente ao Design da Informação ${ }^{3}$, por meio de estudos de desenho pictogramas, infográficos, ilustração informativa e desenho de mapas, interligando-a às disciplinas de Laboratório de Desenho / e III e dando suporte às disciplinas de Prática Projetual de projetos de sinalização, embalagem, design editorial e design digital.

A atividade descrita e analisada nesse estudo foi realizada de forma coletiva, em grupos de quatro a cinco alunos, em dois encontros de quatro (4) horas e consistiu nas seguintes etapas:

- Visualização do filme O desenho conectando conhecimentos. Episódio 6: O desenho no Design de Informação (2018) e discussão sobre a entrevista com Tim Fendley, identificando, coletivamente, as etapas do projeto Legible London;

- Análise de diferentes sistemas de wayfinding, identificando a participação do desenho em seus projetos;

- Escolha de um problema de projeto envolvendo um sistema de wayfinding e incluindo a necessidade do desenho de mapas;

- Identificação dos usuários;

- Identificação dos cenários a partir da identificação de categorias e jornadas dos usuários (frequentes, esporádicos, ocasionais, que usam o transporte público ou transporte próprio - carro ou bicicleta);

- Desenhos de modelos mentais dos mapas a partir dos cenários estabelecidos e posterior análise comparativa com os desenhos técnicos já existentes (diagramas, plantas técnicas, mapas e fotografias via satélite). Esses desenhos foram feitos inicialmente de forma individual e em seguida de forma coletiva;

- Comparação das informações levantadas quanto aos usuários, cenários, modelos mentais, com a elaboração de um painel de informações;

- Estudos de estilos de representação para os desenhos finais (representações esquemáticas, bidimensionais ou tridimensionais);

- Análise coletiva dos desenhos;

2 O filme faz parte de uma série de entrevistas realizadas por Zimmermann (2018) com designers, educadores e pesquisadores do Reino Unido, os quais abordam diferentes percepções do desenho. O material possui fins educativos e está disponível na internet no site www.pesquisaemdesenho.com, com legendas em português.

${ }^{3}$ A ementa e conteúdo dessa disciplina foram elaboradas a partir do modelo de ensino de desenho proposto por Zimmermann (2016). 
- Alterações a partir das análises;

- Estudos finais e concepção das diretrizes visuais para a finalização dos desenhos. Em função do tempo da atividade não foi prevista a produção final dos desenhos. Também foi definido que todos os desenhos deveriam ser feitos de forma manual, considerando as limitações técnicas quanto ao uso de softwares gráficos na $2^{\mathrm{a}}$ fase do curso.

Os locais de aplicação de sistemas de wayfinding selecionados pelos estudantes foram o Campus Itacorubi da UDESC e seus arredores, incluindo os locais e serviços que atendem aos estudantes e público da instituição, bem como o centro histórico de Florianópolis e o terminal central de ônibus de Florianópolis.

\section{Resultados}

Primeiramente, o fato da atividade iniciar a partir de experiências profissionais aplicadas a projetos reais de wayfinding e relatadas visualmente e de forma didática (entrevista em filme) instigou as discussões iniciais sobre o projeto a ser executado. Esta etapa inicial auxiliou na identificação das etapas projetuais e identificação das funções do desenho no decorrer do projeto. Esse material serviu de guia para toda a atividade.

Já a escolha dos locais para desenvolvimento de um sistema de wayfinding mobilizou o engajamento dos alunos na realização da atividade, pela identificação dos alunos participantes como os possíveis usuários dos projetos. Alguns estudantes relataram, durante a atividade, as dificuldades que tiveram em se localizar no Campus quando ingressaram na instituição e identificaram que um projeto de wayfinding poderia auxiliar novos alunos e público externo em situações semelhantes.

$\mathrm{Na}$ etapa de identificação dos usuários, os alunos estabeleceram personas para as principais categorias. Essas categorias variaram tanto pela familiaridade dos usuários com os espaços analisados, quanto pelos meios de transporte que utilizam. Foi utilizado o desenho para a representação das personas (fig. 2).

Figura 2: Estudos usuários/personas - (usados com a permissão dos alunos ${ }^{4}$ ).
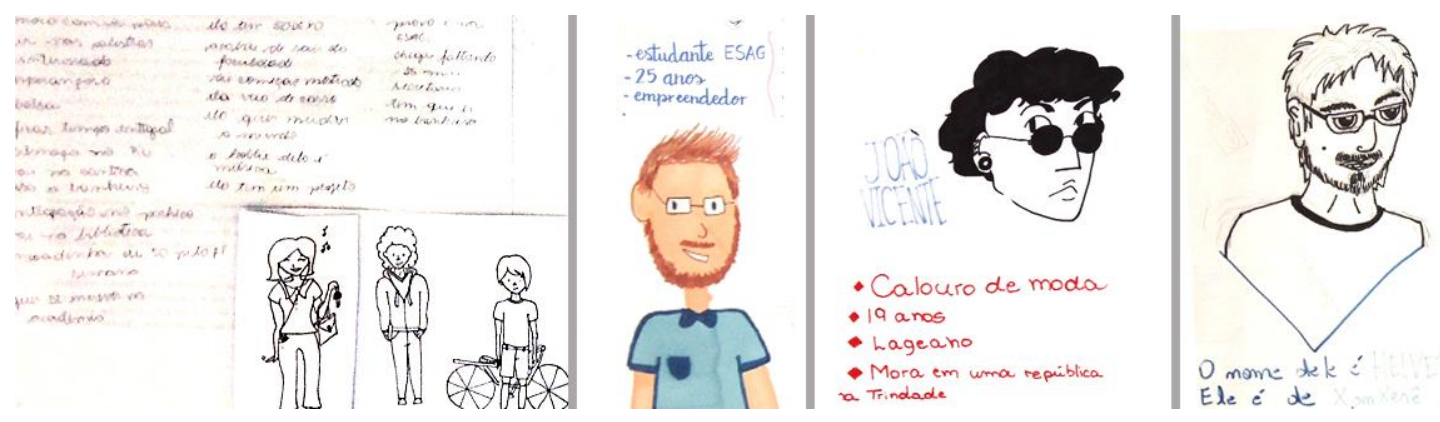

Para a representação das jornadas, os alunos usaram o mesmo modelo de esquema gráfico utilizado no projeto Legible London, o que os auxiliou a identificar os diferentes tipos de percursos dos usuários (fig. 3).

${ }^{4}$ O Termo de Livre Consentimento prevê o anonimato dos alunos participantes. 
Figura 3: Estudos de jornadas dos usuários - (usados com a permissão dos alunos ${ }^{5}$ ).

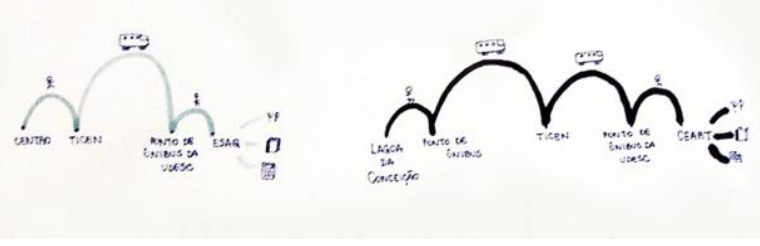

Para a elaboração dos paineis de informações, cada grupo de alunos pode usar uma das paredes da sala. Esse paineis auxiliaram aos alunos para que pudessem observar o conjunto dos dados coletados e complexidade das informações e pudessem discutir coletivamente.

Quanto à etapa de desenho dos modelos mentais dos espaços estudados e posterior comparativo com desenhos técnicos já existentes, os alunos identificaram grande variação entre as dimensões, proporções e localização de espaços e ambientes 'imaginados' e 'reais', causando grande surpresa entre os participantes. Essa variação pode ser observada nos esboços apresentados na figura 4 a seguir. Assim, a partir dessa experiência, os alunos identificaram a importância do contato com os usuários, suas experiências, percepções, familiaridade e objetivos quanto aos espaços envolvidos no projeto, bem como a relevância do desenho de esboços como forma de pensar pelo desenho, organizar ideias, compartilhar informações e planejar ações.

Figura 4: Estudos de modelos mentais do Campus Itacorubi da UDESC - (usados com a permissão dos alunos ${ }^{6}$ ).
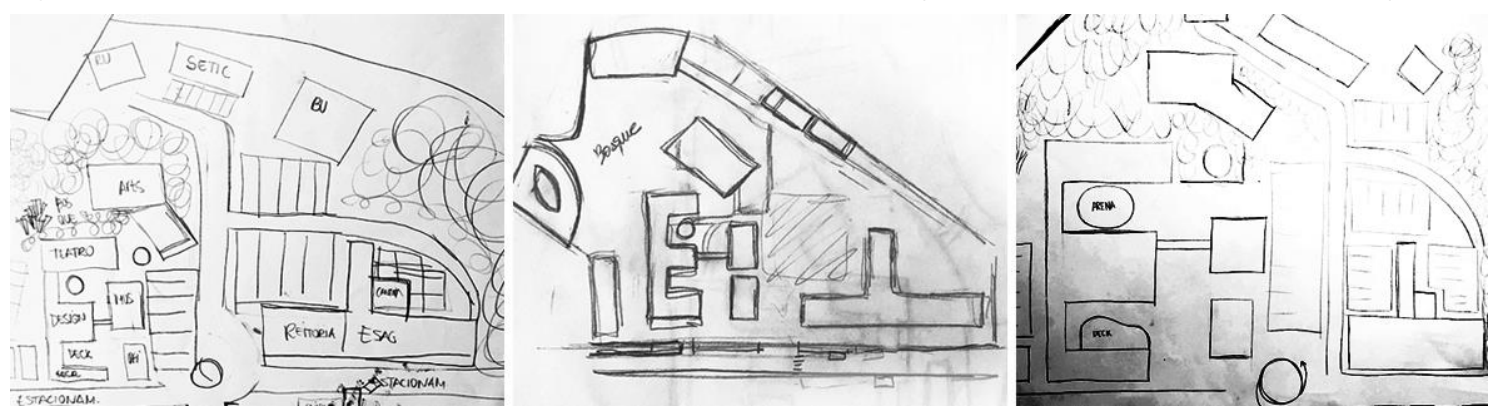

Já, quanto aos estudos de diretrizes visuais para a representação final dos mapas, os alunos observaram que a função estética não pode se sobressair em relação a função informativa, ou melhor, as escolhas estéticas devem servir à organização das informações. Entretanto, são diversas as possibilidades de linguagem estética na elaboração de mapas, e cabe ao designer identificar quais são as mais adequadas aos seus usuários, mensagem e objetivos. Na figura 5 são apresentados diferentes exemplos de linguagem visual elaboradas pelos alunos, com variação de técnica, paleta cromática, composição e forma de representação (bidimensional e tridimensional).

5 O Termo de Livre Consentimento prevê o anonimato dos alunos participantes.

6 O Termo de Livre Consentimento prevê o anonimato dos alunos participantes. 
Figura 5: Estudos de diretrizes visuais para a representação final dos mapas (usados com a permissão dos alunos).

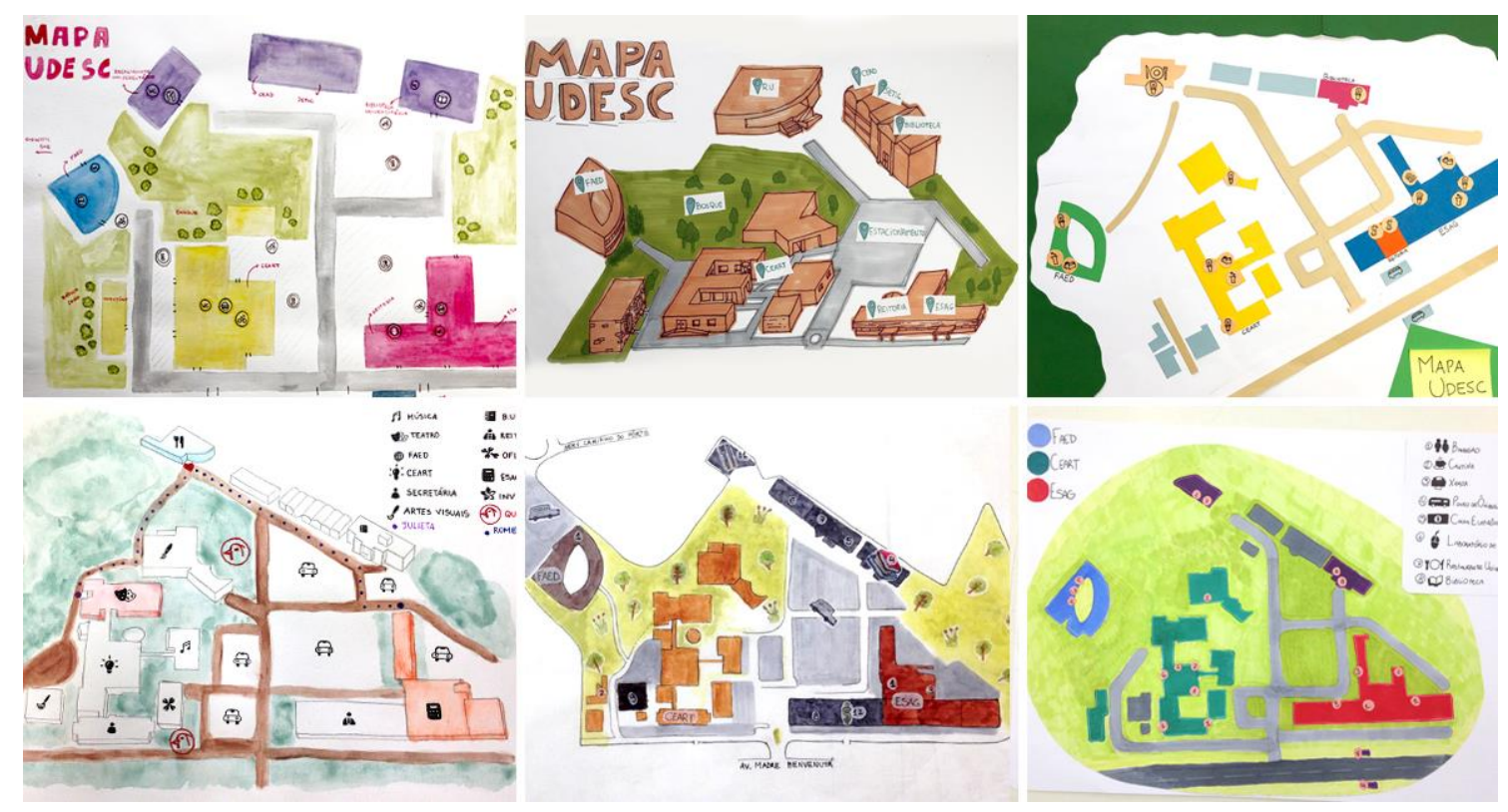

Na etapa de análise coletiva, os alunos puderem contribuir com os diferentes projetos. Essas sugestões foram assinaladas nos desenhos em papeis de outras cores (fig. 6). Essa etapa foi importante tanto pela análise crítica do resultado, como pelo exercício do trabalho coletivo em desenho.

Figura 6: Análise coletiva com sugestões para alterações (usados com a permissão dos alunos).

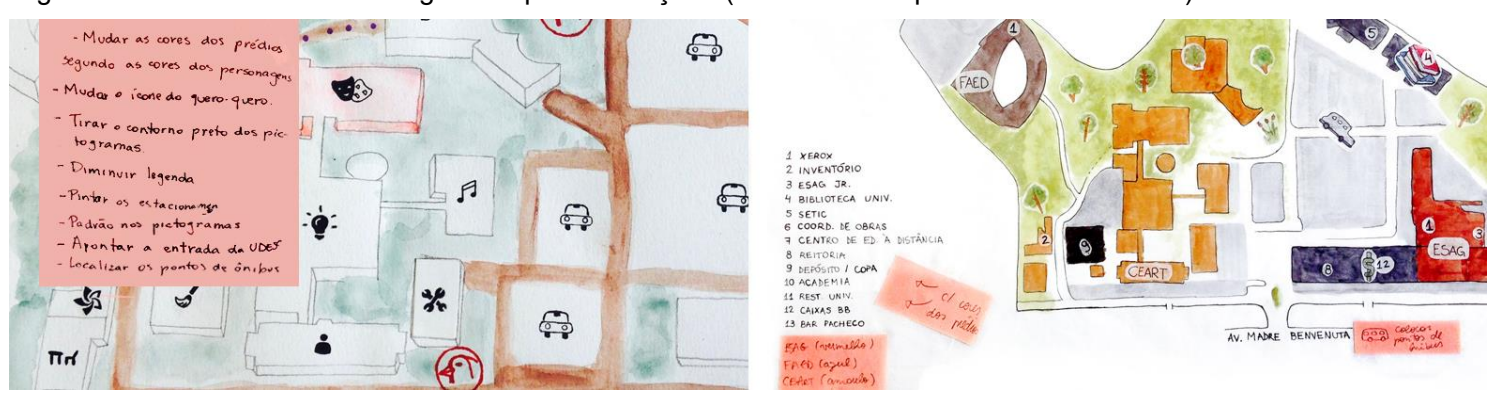

Os alunos também identificaram a necessidade do uso de pictogramas em alguns dos mapas produzidos, suscitando a possibilidade de estudo desse conteúdo sequencialmente ou como integrante do projeto ${ }^{7}$.

Quanto aos conteúdos referentes a aspectos técnicos do desenho, foram trabalhados: a compreensão de espaços tridimensionais pelo desenho, a representação desses espaços e interlocução entre espaços tridimensionais, suas representações bidimensionais e seus usuários, síntese gráfica, composição, proporção, escala, noção espacial, representação bidimensional e tridimensional e experimentação de materiais, como caneta marcador, caneta nanquim, aquarela e colagem.

${ }^{7} \mathrm{O}$ desenho de pictogramas havia sido trabalho em uma atividade anterior, aplicada a outro projeto. 
Por fim, identificou-se a necessidade de ajustes quanto ao tempo de projeto em experiências futuras, visto que a atividade inclui não apenas a execução de tarefas de caráter técnico, como também a pesquisa, análise e a interpretação dos dados que compõem o projeto. Com base nesses aspectos é importante a escolha de projetos de baixa complexidade projetual para a sua execução, visto que o objetivo da atividade se refere ao ensino e prática do desenho.

\section{Conclusões e considerações finais}

Observou-se que o modelo cumpriu com seus objetivos e sua aplicação indicou que pode ser adaptado a diferentes situações de ensino do desenho, podendo ser direcionado ao estudo do processo do desenho em projetos, à compreensão de espaços tridimensionais pelo desenho, à representação desses espaços e interlocução entre espaços tridimensionais, suas representações bidimensionais e seus usuários, além dos aspectos técnicos, como síntese gráfica, composição, proporção, escala, noção espacial, representação bidimensional e tridimensional e experimentação de materiais.

Também se identificou o design da informação, mais especificamente sistemas de wayfinding, com um tema capaz de gerar conteúdo diversos a serem explorados em disciplinas de ensino do desenho em Design Gráfico. Além disso, a aplicação do modelo proposto permitiu a identificação de uma aproximação entre disciplinas do currículo, dando suporte a Práticas Projetuais referentes especificamente a projetos de sinalização, bem como permitiu aproximar o exercício do desenho ao Design da Informação, ampliando a compreensão desse campo do design junto aos participantes.

\section{Referências}

Adams, E. (2009). Drawing: a tool for design. Lancing: Power Drawing/The Campaign for Drawing.

Cabau, P. (2011). Design pelo desenho: exercícios, jogos, problemas e simulações. Lisboa: FCA Design.

Cross, N. (2011). Design thinking. London: Bloomsbury Academic.

Farthing, S. (2011). The Bigger Picture of Drawing. In: Kantrowitz, A.; Brew, A.; Fava, M. (Eds.) Thinking through drawing: practice into knowledge. Proceedings of an interdisciplinary symposium on drawing, cognition and education (pp. 21-25). New York: Teachers College, Columbia University. Retrieved November 18, 2014.

Goel, V. (1995). Sketches of thought. Cambridge MA: MIT Press.

Goldschmidt, G. (1991). The dialectics of sketching. Creativity Research Journal. v. 4, 1991, p. 123-143.

O desenho conectando conhecimentos. Episódio 6: O desenho no Design de Informação: Tim Fendley, Applied Wayfinding. 2018. Entrevista com Tim Fendley. Direção e Produção: Anelise Zimmermann. Londres/Florianópolis, Editora UDESC, 2018. 1 DVD (20 min), MP4, color. Português/Inglês. In: Home: Pesquisaemdesenho. $<$ https://www.pesquisaemdesenho.com/episodio-6>.

Schaeverbeke, R. \& Heylighen, A. (2012). Drawing and designing as a research approach to rethink teaching. Theory by design: Architectural research made explicit in the design teaching studio. Bélgica, Faculty of Design Sciences, University of Antwerp, 2012, p. 159-166.

Twyman, M. L. (1979). A schema for the study of graphic language. In: Paul A. Kolers, Merald E. 
Wrolstad \& Herman Bouma (Eds.). Processing of visible language. Nova York \& Londres: Plenum Press, vol.1, p. 117-150.

Twyman, M. L. (1982). The graphic presentation of language. Information Design Journal, v.3, n.1, p. 2-22.

Twyman, M. L. (1985). Using pictorial language: a discussion of the dimensions of the problem. In: DUFTY, Thomas \& WALLER, Robert. (Eds.) Designing usable texts. Orlando, Flórida: Academic Press, p. 245-312.

Sless, D. (1992). What is Information Design? in: Robyn Penman \& David Sless (Eds.), proceedings of the Symposium Designing information for people, p. 1-16.

Zimmermann, A. (2016). O ensino do desenho na formação em Design Gráfico: uma abordagem projetual e interdisciplinar. Tese de doutorado. Programa de Pósgraduação em Design. Universidade Federal de Pernambuco, Recife.

Zimmermann, A. \& Coutinho, S. G. (2018). Teaching drawing based on the design process: exploring creativity. The Fifth International Conference on Design Creativity (ICDC2018), Bath, 2018, p. 306-313.

\section{Sobre o(a/s) autor(a/es)}

Anelise Zimmermann, Doutora, UDESC, Brazil <anelise.zimmermann@udesc.br>

Solange G. Coutinho, PhD, UFPE, Brazil <sol2015ufpe@gmail.com> 